\title{
A Case of Temporal Bone Cholesterol Granuloma Extending to Posterior Cranial Fossa without Middle Ear Involvement
}

\author{
Min Chul Ko ${ }^{1}$, Ji Yun Jeong ${ }^{2}$, Sung Kyoo Hwang ${ }^{3}$, and Myung Hoon Yoo $^{4}$ \\ ${ }^{1}$ Department of Otorhinolaryngology-Head and Neck Surgery, School of Medicine, Kyungpook National University, \\ Kyungpook National University Hospital, Daegu; and \\ ${ }^{2}$ Departments of Pathology, ${ }^{3}$ Neurosurgery, ${ }^{4}$ Otorhinolaryngology-Head and Neck Surgery, School of Medicine, \\ Kyungpook National University, Kyungpook National University Chilgok Hospital, Daegu, Korea
}

\author{
중이 병변 없이 후두개와로 확장된 측두골 콜레스테롤 육아종 1 예 \\ 고민철 ${ }^{1}$ 정지윤 ${ }^{2} \cdot$ 황성규 $^{3} \cdot$ 유명훈 $^{4}$ \\ 경북대학교 의과대학 경북대학교병원 이비인후과학교실, ${ }^{1}$ \\ 경북대학교 의과대학 칠곡경북대학교병원 병리학교실, ${ }^{2}$ 신경외과학교실, ${ }^{3}$ 이비인후과학교실 ${ }^{4}$
}

\author{
Received September 23, 2019 \\ Revised November 25, 2019 \\ Accepted December 9, 2019 \\ Address for correspondence \\ Myung Hoon Yoo, MD \\ Department of Otorhinolaryngology- \\ Head and Neck Surgery, \\ School of Medicine, \\ Kyungpook National \\ Chilgok University Hospital, \\ 807 Hoguk-ro, Buk-gu, \\ Daegu 41404, Korea \\ Tel +82-53-200-5777 \\ Fax +82-53-200-2027 \\ E-mail cooleo312@gmail.com
}

Cholesterol granulomas of the temporal bone can occur in the petrous apex, middle ear, and mastoid cavity. Although cholesterol granulomas in the petrous apex often extend to the middle cranial fossa, cholesterol granulomas in the middle ear and mastoids rarely invade the cranial cavity with bony erosion. We report a case of a large cholesterol granuloma involving the posterior cranial fossa with pneumatic mastoid and not affecting the middle ear. The patient had no otologic or neurologic symptoms, and the mass was found incidentally on a brain MRI included in a regular medical checkup. The mass was removed via a combined transmastoid and suboccipital approach without complications, and characteristic pathology findings demonstrated a cholesterol granuloma. Korean J Otorhinolaryngol-Head Neck Surg 2020;63(7):324-9

Key Words Cholesterol · Granuloma Posterior cranial fossa - Temporal bone.

\section{Introduction}

Cholesterol granulomas can occur anywhere in the body, with the most common site being the temporal bone." Cholesterol granulomas are often associated with otitis media and are characterized by a blue tympanic membrane on otoscopic examination. ${ }^{2}$ Cholesterol granulomas frequently invade the middle ear or petrous apex and can lead to hearing impairments; ${ }^{2)}$ however, a cholesterol granuloma that extensively

This is an Open Access article distributed under the terms of the Creative Commons Attribution Non-Commercial License (https://creativecommons.org/licenses/by-nc/4.0) which permits unrestricted non-commercial use, distribution, and reproduction in any medium, provided the original work is properly cited. destroys the bone structure and invades the cranium is uncommon, ${ }^{1)}$ and cholesterol granuloma cases extending from the mastoid cavity to the cranial cavity without lesions in the middle ear or petrous apex are rarely discussed in the literature. We report on a case of a temporal bone mass extending into the cranial cavity that was found incidentally without accompanying otologic signs or symptoms and was confirmed as a cholesterol granuloma postoperatively.

\section{Case}

A 31-year-old male visited our clinic, presenting with a sus- 
picious mass lesion in the right temporal bone that had been found incidentally on a brain MRI during a routine health checkup. The patient reported being in a traffic accident three months before, but there was no serious head trauma and no other relevant medical history. On physical examination, both eardrums were intact, and no otologic symptoms such as hearing loss, tinnitus, otalgia, or otorrhea were observed. Brain MRI imaging revealed a $3.5 \mathrm{~cm}$ soft tissue mass extending from the right mastoid process to the posterior cranial fossa. The mass demonstrated high signal intensity on T1-weighted images and high signal intensity mixed with partial iso-signal intensity on T2-weighted images. The mass was confined to the epidural space, and no evidence of brain parenchymal infiltration was observed (Fig. 1). A brain CT scan revealed a non-enhancing mass lesion with focal calcification at the posterior fossa, with expansion and remodeling of the outer table,
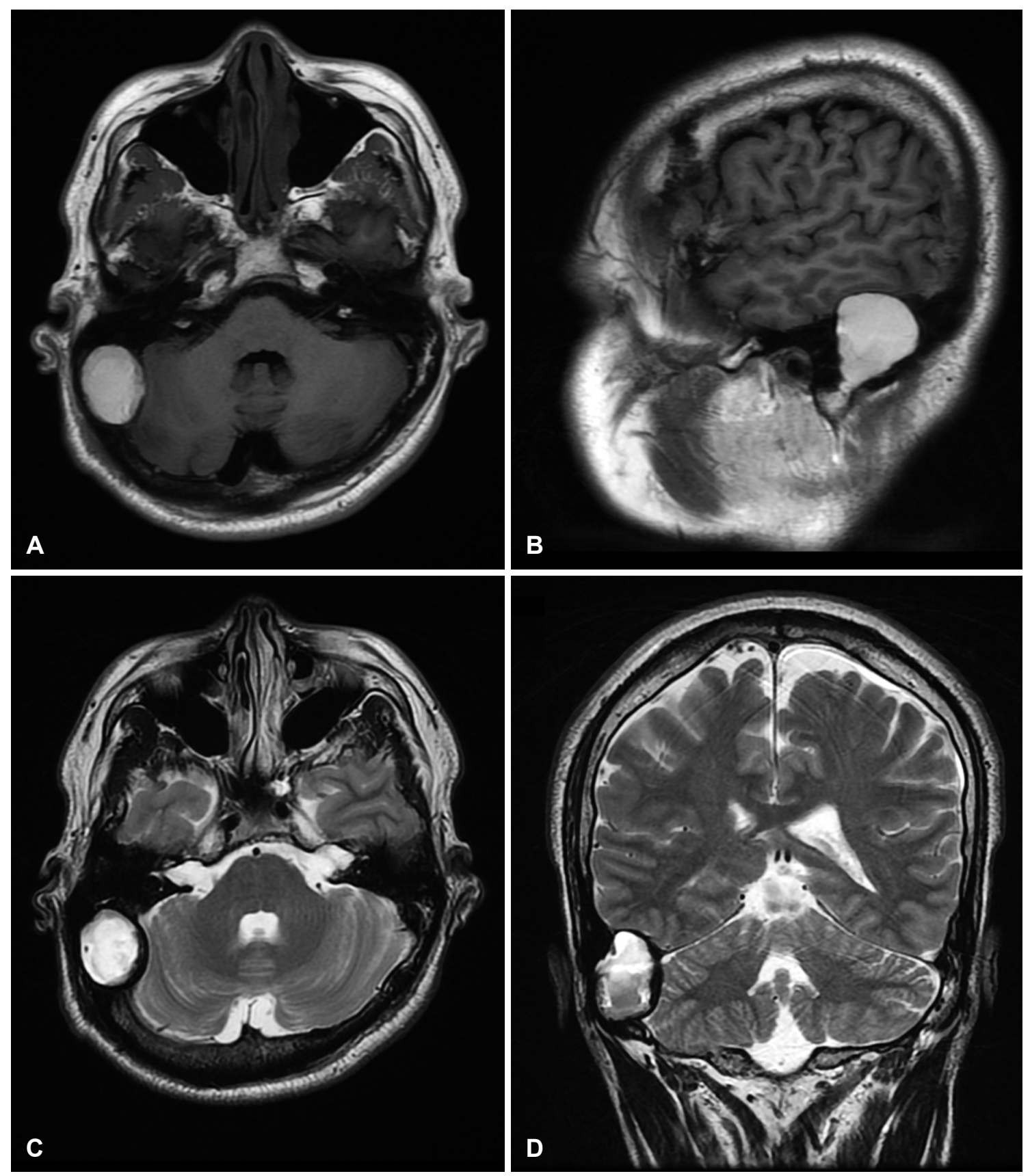

Fig. 1. Preoperative MRI findings. Mass showing high signal intensity on T1-weighted axial (A) and sagittal images (B), and high signal intensity on T2-weighted images (C), including partial iso-signal intensity (D). Images show an expansile mass in the right temporal bone extending into the posterior cranial fossa. 
erosion of the inner table, focal bony defect with a thin wall in mastoid air cell, and compression of the sigmoid sinus to the medial side (Fig. 2).

We performed a mass excision via transmastoid and suboccipital craniotomy in cooperation with the neurosurgery department. A simple mastoidectomy was done and there was no abnormality in the mastoid air cells. Only the outer part of the skull bone remained, and the inner part was defective. Through the skull defect, the dura mater of the middle cranial fossa and posterior cranial fossa was exposed (Fig. 3A). The cyst wall was thick and very tightly adherent to the underlying dura mater and sigmoid sinus. The cyst was filled with brown col- ored cystic fluid along with semisolid components including a glistening material (Fig. 3B). The cystic and semisolid components in the cyst were evacuated. No evidence of involvement of the lesion in the middle ear or brain parenchyma was observed. A small tearing occurred in the transverse sinus during cyst wall dissection but was corrected uneventfully. The defect in the extradural area was filled with abdominal fat, and the craniotomy site was reconstructed using a temporal bone outer table bony fragment and miniplate (Fig. 3C).

Postoperative pathologic findings revealed a lack of definite epithelium. Cholesterol cleft, focal chronic inflammation, and hemosiderin laden macrophages were identified (Fig. 4), con-
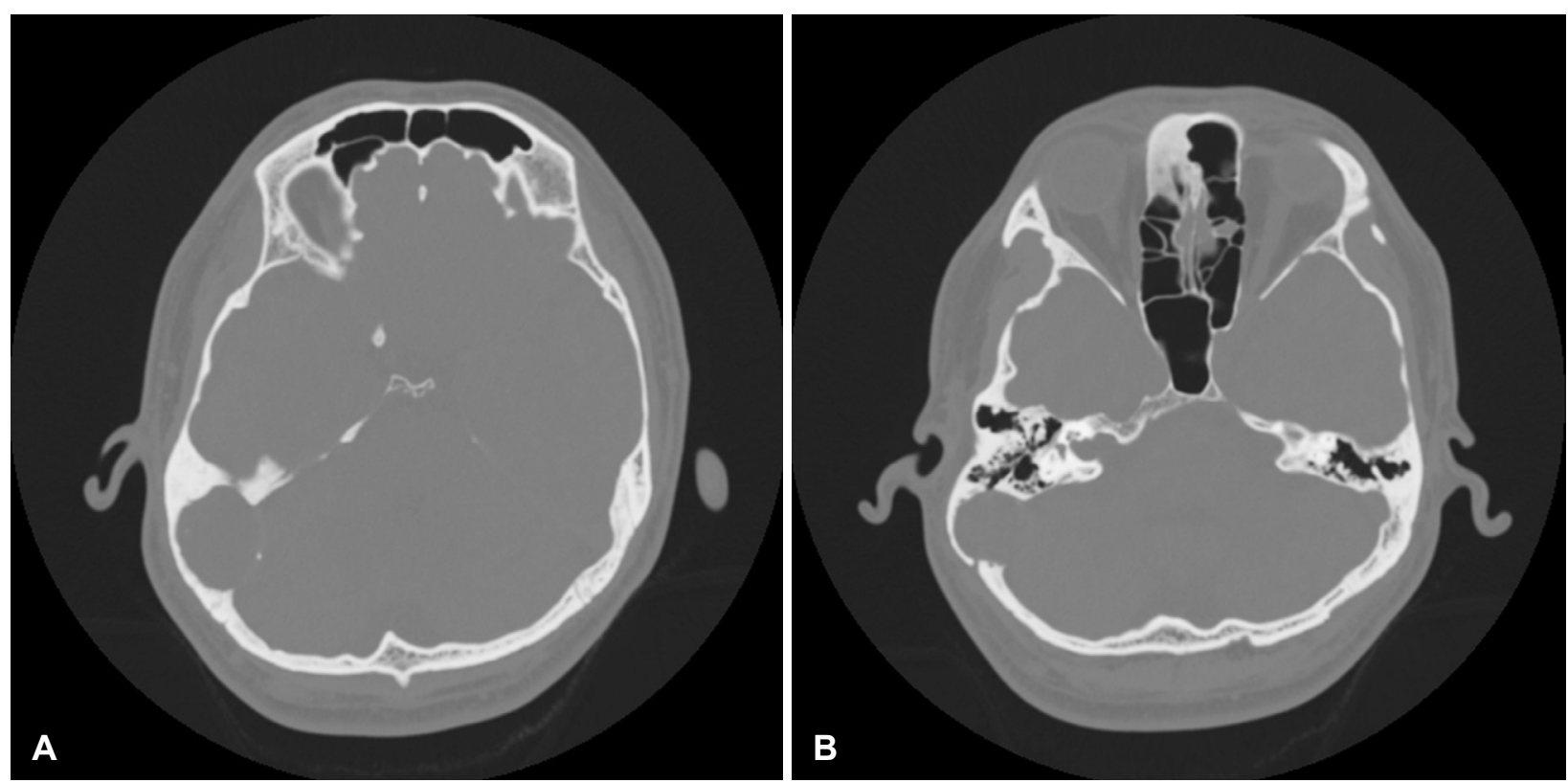

Fig. 2. Preoperative findings of brain CT. Mass destruction of the temporal bone inner table and expansion into posterior cranial fossa (A). Brain CT showing pneumatic middle ear and mastoid cavity (B).
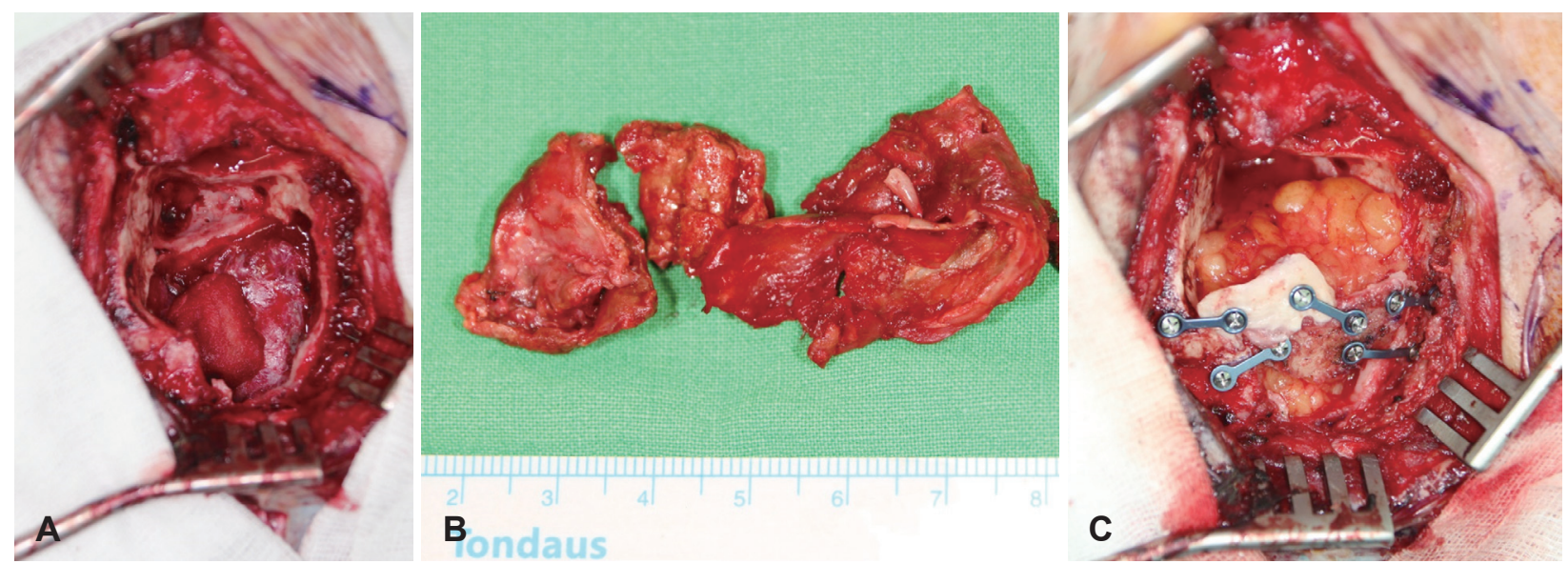

Fig. 3. Gross and surgical findings. Dura mater of the posterior cranial fossa exposed after removal of the cystic mass (A). Findings showing thick cystic walls (B). Craniotomy site was reconstructed using abdominal fat and temporal bone chips (C). 
sistent with pathologic findings indicating a cholesterol granuloma. The postoperative period was uneventful. No evidence of recurrence was observed on a temporal bone CT at three months postoperatively nor on a brain MR at six months after surgery (Fig. 5). No hearing deterioration or other surgeryassociated symptoms were observed over the one-year followup period.

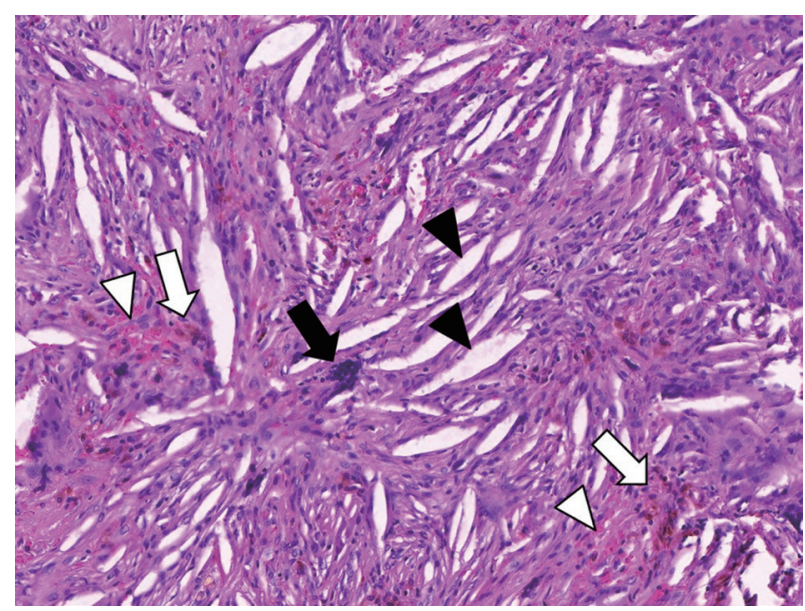

Fig. 4. Histopathologic findings. Results showing several cholesterol clefts (black arrowheads) surrounded by inflammatory cells. Images also show some giant cells with bunched nuclei (black arrow) and a mixture of brown colored hemosiderin pigment and hemosiderin-laden histiocytes (white arrows). RBCs (white arrowheads) partially scattered into the extravascular tissue (hematoxylin \& eosin staining, $\times 100)$.

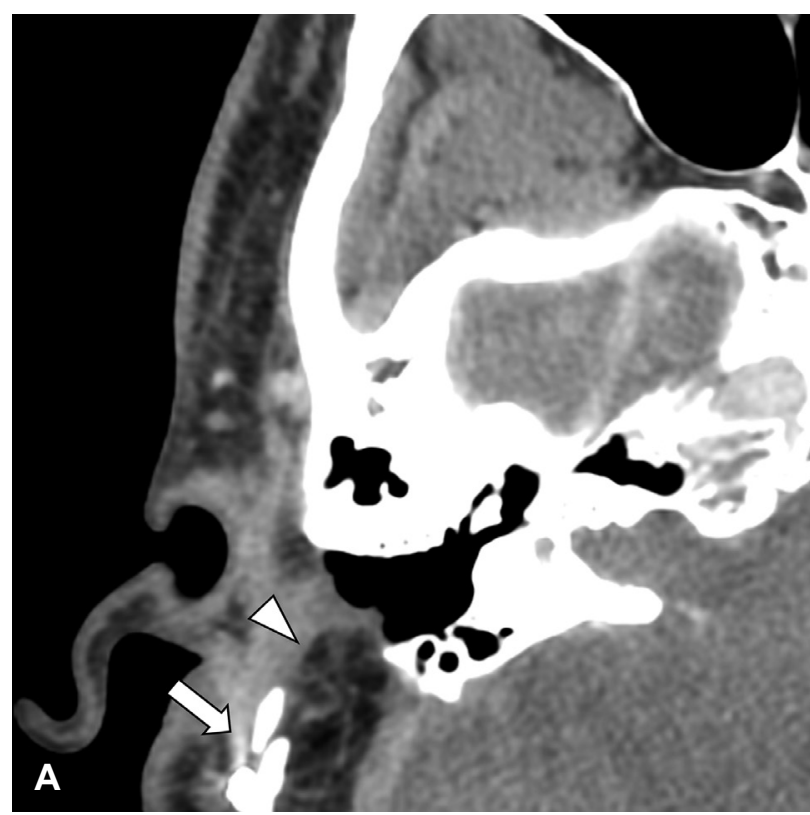

\section{Discussion}

The most common site of a cholesterol granuloma is the temporal bone, and this lesion can present in the petrous apex, middle ear, or mastoid region. Most cases of invasion into the cranial fossa are cholesterol granulomas in the petrous apex, and cranial involvement of the middle ear and mastoid region are infrequently observed. ${ }^{3-5)}$ The case discussed in this report is quite rare, as unlike in previous cases, no otologic symptoms nor middle ear involvement were observed. Conductive hearing loss can occur in patients with cholesterol granulomas in the middle ear, and cranial nerve impairment, including sensory neural hearing loss and facial palsy, can accompany cholesterol granulomas in the petrous apex. ${ }^{2)}$ Involvement of the cranial fossa may cause neurologic symptoms such as headache, insomnia, and abnormal behavior. ${ }^{2,3,5)}$ Considering the known aggressive progression of the disease, we presumed that these neurological symptoms would have occurred if the mass was left untreated. Our patient was treated prior to the onset of symptoms and recovered without complications.

Macroscopically, a cholesterol granuloma typically presents as a dark brown cyst due to hemosiderin deposition, although it can also have a yellowish color when involving adipose tissue, and demonstrates shiny properties due to cholesterol crystals. ${ }^{6)}$ Our patient's mass presented with typical gross findings. Martin, et al. ${ }^{7)}$ have suggested that differences in gross findings could occur between aggressive and benign

Fig. 5. Postoperative findings. Temporal bone CT (three months postoperative) (A) and brain MR gradient echo T2 scan (six months postoperative) (B). No evidence of recurrence in either evaluation. Bony fragment, miniplate (white arrow), and abdominal fat (white arrowheads) used for intraoperative reconstruction. 
cases, ${ }^{7)}$ explaining that in benign cases, cholesterol-rich body fluids fill the potential space, while in aggressive cases, cysts present with thicker walls due to inflammatory reactions. Surgical findings in our patient revealed thick cystic walls similar to gross findings of previously reported aggressive cholesterol granuloma cases. ${ }^{8-10)}$ Kuruma, et al. ${ }^{8)}$ have presented several potential reasons for the aggressive expansion of certain cholesterol granulomas, indicating that negative pressure caused by drainage failure can lead to bleeding from the mucosal blood vessels and that coaptation of the marrow and mucous membranes due to exposure of the bone marrow can also lead to bleeding. In the latter case, a cholesterol granuloma from the marrow-rich petrous apex often extends into the cranial fossa. Authors also suggested a robust blood supply, such as the sigmoid sinus or carotid artery, could be a driving force for progressive cyst expansion. ${ }^{8,11}$ In our patient, this last theory is presumed to apply to this case as the mass was found tightly adherent to the sigmoid sinus.

Oh, et al. ${ }^{1)}$ reported a case of cholesterol granuloma in the right middle cranial fossa incidentally found on a temporal CT scan following suspicion of left chronic otitis media; this patient presented with trauma history but without chronic otitis media in the right ear. This case differed from ours in that it was accompanied by conductive hearing loss on the right side, and the mass filled the epitympanum with a tegmen defect, suggesting extension from mastoid. ${ }^{1)}$ In our case, an aggressive cholesterol granuloma in the posterior fossa was found without the presence of middle ear lesions or common symptoms such as hearing loss, tinnitus, or the characteristic blue eardrum. The patient had been in a traffic accident three months before surgery that could have led to minor bleeding; this might have been a trigger of cholesterol granuloma, but there was no direct evidence that suggested the accident was the main cause of the lesion.

Complete resection of the granuloma in the middle ear is not always necessary because the purpose of treatment is to enable proper drainage and ventilation, and conservative treatment, such as insertion of a ventilation tube, can be sufficient. ${ }^{2)}$ However, cholesterol granulomas with petrous apex involvement, intracranial involvement, or aggressive tendencies usually require surgical resection. The method of surgical treatment depends on the location of the cholesterol granuloma, the hearing status of the patient, and other accompanying symptoms. ${ }^{2,12)}$ In our case, we exposed the mass via a transmastoid and suboccipital approach for complete resection, and it was removed without serious complications. In previous cases of cholesterol granuloma with intracranial involvement, some have reported re-pneumatizing the temporal bone through drainage and ventilation of the cyst, ${ }^{1)}$ although some of these cases also reported cholesterol granuloma recurrence. ${ }^{2,3)}$ In most cases, complete resection of the cyst was performed. . $^{3-5,8,10,13)}$ Complications, such as bleeding and dura injury due to excessive dissection, can occur necessitating meticulous surgical technique. Our patient had slight bleeding due to mass adhesion to the sigmoid sinus, but resection was successful, and there were no major issues. Although surgical resection is thought to prevent recurrence, surgical methods should be selected while considering possible surgical complications.

Along with previous case reports, this paper describes the differences between a common cholesterol granuloma in the middle ear cavity and an aggressive cholesterol granuloma. Typical cholesterol granulomas are surrounded by a thin, fibrous lining, but aggressive cholesterol granulomas extending into the cranial cavity present with measurably thicker walls. ${ }^{1,8,10,13)}$ In most of these cases, adhesion to the dura or blood vessels was observed during surgery, ${ }^{1,4,5,8)}$ however, the consensus is that complications such as bleeding or dural injury can be reduced if such findings are kept in mind. Although the present patient was asymptomatic, evidence suggests that temporal bone cholesterol granulomas can cause neurological symptoms such as dizziness, headache, cranial nerve impairment, and abnormal behavior without ear symptoms. Some of the characteristics of cholesterol granulomas, including lesions of the cranial fossa, have been identified in previous cases, but more studies are needed due to their relatively rare incidence.

\section{Acknowledgments}

None.

\section{Author Contribution}

Conceptualization: Myung Hoon Yoo. Data curation: Min Chul Ko. Investigation: Min Chul Ko, Myung Hoon Yoo. Resources: Ji Yun Jeong, Sung Kyoo Hwang. Supervision: Myung Hoon Yoo. Visualization: Sung Kyoo Hwang. Writing — original draft: Min Chul Ko. Writing — review \& edit: Ji Yun Jeong, Sung Kyoo Hwang, Myung Hoon Yoo.

\section{ORCID}

Myung Hoon Yoo https://orcid.org/0000-0002-9158-8924

\section{REFERENCES}

1) Oh JK, Yang SD, Moon SY, Shin SO. A case of intracranial and extradural cholesterol granuloma. Korean J OtorhinolaryngologyHead and Neck Surg 2009;52(11):913-6.

2) Goldofsky E, Hoffman RA, Holliday RA, Cohen NL. Cholesterol cysts of the temporal bone: Diagnosis and treatment. Ann Otol Rhinol Laryngol 1991;100(3):181-7. 
3) Brodie SW, Chaurasia MK. A rare intracranial complication of cholesterol granuloma. J Laryngol Otol 1985;99(5):491-5.

4) Morioka T, Fujii K, Nishio S, Miyagi Y, Nagata S, Hasuo K, et al. Cholesterol granuloma in the middle cranial fossa: Report of two cases. Neuroradiology 1995;37(7):564-7.

5) Muzumdar DP, Goel A, Desai K, Chagla A. Cholesterol granuloma in the middle fossa presenting 30 years after surgery for chronic otitis media: A case report. J Clin Neurosci 2002;9(4):447-50.

6) Nager GT, Vanderveen TS. Cholesterol granuloma involving the temporal bone. Ann Otol Rhinol Laryngol 1976;85(2 pt 1):204-9.

7) Martin TP, Tzifa KT, Chavda S, Irving RM. A large and uncharacteristically aggressive cholesterol granuloma of the middle ear. J Laryngol Otol 2005;119(12):1001-3.

8) Kuruma T, Tanigawa T, Uchida Y, Tetsuya O, Ueda H. Large cholesterol granuloma of the middle ear eroding into the middle cranial fossa. Case Rep Otolaryngol 2017;2017:4793786.
9) Palva T, Lehto VP, Johnsson LG, Virtanen I, Mäkinen J. Large cholesterol granuloma cysts in the mastoid. Clinical and histopathologic findings. Arch Otolaryngol 1985;111(12):786-91.

10) Polo R, Medina M, Labatut T, Alonso A, Vaca M. Mastoid cholesterol granuloma with posterior cranial fossa compression. Otol Neurotol 2013;34(7):e103-4.

11) Pfister MH, Jackler RK, Kunda L. Aggressiveness in cholesterol granuloma of the temporal bone may be determined by the vigor of its blood source. Otol Neurotol 2007;28(2):232-5.

12) Rinaldo A, Ferlito A, Cureoglu S, Devaney KO, Schachern PA, Paparella MM. Cholesterol granuloma of the temporal bone: A pathologic designation or a clinical diagnosis? Acta Otolaryngol 2005;125(1):8690.

13) Jang $\mathrm{CH}$, Cho YB. Huge cholesterol granuloma of the middle ear extending to middle cranial fossa. In Vivo 2009;23(1):191-3. 